\title{
Application of Real-Time Voltage Security Assessment to the Hellenic Interconnected System
}

\author{
T. Van Cutsem
}

FNRS, Dept. of Electrical Engineering and Computer Science (Montefiore Institute), University of Liège, Sart Tilman B37, B-4000 Liège, Belgium, t.vancutsem@ulg.ac.be

\section{J. Kabouris, G. Christoforidis}

Hellenic Transmission System Operator, 11 Amphitheas Ave., N. Smirni, Athens, Greece

C.D. Vournas

School of Electrical and Computer Eng., National Technical University of Athens, Iroon Polytechniou 9, Zografou, GR-15773 Athens, Greece, vournas@power.ece.ntua.gr

\begin{abstract}
This paper describes the on-line Voltage Security Assessment methods implemented at the national control center of the Hellenic Interconnected System, within the framework of the OMASES project funded by the European Union. The heart of all computations is a fast time-domain method. Security is analysed with respect to power transfers in critical corridors or power consumption in load areas. Results take on the form of either pre-contingency secure operation limits, or post-contingency loadability limits, together with various diagnosis tips. Contingency filtering is performed to meet the real-time requirement. Typical results obtained during the test phase of the project are reported.
\end{abstract}

\section{Keywords}

Voltage Stability, Voltage Security Assessment, Quasi steady-state simulation, Secure Operation Limits, Loadability limits. 


\section{Introduction}

Voltage instability is widely recognised as a significant threat of power system blackout [1]. As far as real-time operation is concerned, there is a need for appropriate tools to identify dangerous contingencies, assess security margins and suggest corrective actions [2, 3].

Over the last decade, efforts have been directed towards making Voltage Security Assessment (VSA) methods compatible with the requirements of on-line applications (computational speed, data maintenance, significance of displayed results, etc.). Examples of such developments are given in Ref. [4], which describes the general practice and gives a sample of existing tools, while Ref. [5] presents techniques for the determination of stability limits.

This paper describes and reports on the application of the on-line VSA function developed within the framework of the OMASES project, sponsored by the European Union. The latter aimed at developing an on-line platform for dynamic security assessment, training simulation and market simulation. The overall project is presented in [6], whereas transient stability assessment results, as well as VSA tests on another system are reported in [7]. This paper focuses on long-term voltage stability driven by load restoration through tap changers, overexcitation limiters, switched compensation and possibly secondary voltage control $[1,8]$.

The Hellenic Interconnected System is voltage stability constrained (especially during summer peak) in the North-South power transfer [9]. In particular, since the local generation in the Athens area is significantly expensive, it is usually driven out of the market by the everyday bidding procedure. In case of reduced security margins, this generation is put in operation (must-run units) in order to alleviate transmission constraints and increase security. Since the operation of expensive units increases the uplifts in the electricity market, it is a major concern for HTSO to avoid such practices and try to operate the system closer to its limits. On-line VSA is the most appropriate tool to achieve this task.

The methods used in the so-called OMASES-VSA module are described in Section 2 and further discussed in Section 3. Their practical implementation is outlined in Section 4. A sample of results stemming from its use at the National Control Center of the Hellenic Transmission System Operator (HTSO) is presented in Section 5. Concluding remarks are offered in Section 6.

\section{Voltage Security Assessment Methods}

\subsection{Quasi steady-state simulation : principle and modelling}

The heart of all computations performed within OMASES-VSA is Quasi Steady-State (QSS) simulation, a fast time-domain method well suited to the analysis of long-term voltage stability phenomena [8].

The QSS approximation relies on time-scale decomposition. The essence of this method is that faster phenomena are represented by their equilibrium conditions instead of their full dynamics. This greatly reduces the complexity of the resulting model and hence provides the computational efficiency required to meet the constraints of an on-line application. In addition, the amount of additional data required by the QSS model is moderate, so that data collection, validation and maintenance is not a big issue. 


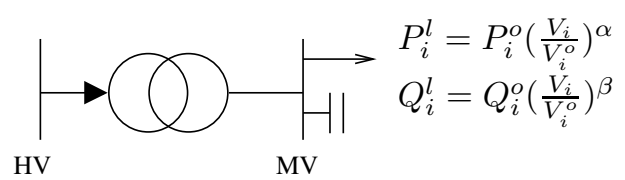

Figure 1: Typical load model

This method, which has been validated with respect to detailed time simulation offers better accuracy and richer interpretations than simple methods based on load flow equations. For instance, in unstable cases, the area in trouble is automatically pointed out, while sensitivity-based diagnosis can be performed on the unstable system trajectory in order to identify appropriate remedial actions. Even when the QSS model involves components and phenomena that could be also accounted for in load flow computations, QSS simulations offer advantages over the latter, while preserving computational efficiency.

Under the QSS approximation, the short-term dynamics of a synchronous generator, its governor and its Automatic Voltage Regulator (AVR), are replaced by three nonlinear algebraic equations [8]. The latter account for the generation saturation, the AVR steady-state gain and the speed droop. These nonlinear equations are solved at each time step, together with the network ones.

For each load, voltage dependent active and reactive powers are assumed at the MV bus behind the HV-MV distribution transformer, in parallel with a shunt compensation capacitor. Studies are still under progress to improve the modelling of loads. The initial tests reported in this paper have been performed with an exponential model, as shown in Fig. 1. Different exponents are used according to the type of load. Load power restoration mainly comes from the Load Tap Changers (LTCs) operating in the HV-MV transformers.

QSS simulation reproduces the long-term dynamics of LTCs, OverExcitation Limiters (OELs), automatically switched shunt compensation, secondary voltage control (if any), protecting devices, etc. This simulation takes into account the (initial and subsequent) delays in between transformer tap changes, the delays before a synchronous machine is switched under constant field current, etc.

\subsection{System stress}

Voltage security is analysed in terms of power transfers, referred to as system stress. The stress is a change in load and generation which makes the system weaker by increasing power transfer over relatively long distances and/or by drawing on reactive power reserves. A system stress is characterised by its direction, i.e. by the participation of each load active and reactive power to the overall load increase and that of each generator active power production to the overall generation adjustment.

VSA is performed at the current system state, provided by the EMS state estimator, and referred to as base case in the remaining of the paper.

Two approaches have been followed for assessing the impact of contingencies, as explained in the next two sections. 


\subsection{Loadability limits}

A loadability limit indicates how much the system can be stressed before reaching instability. To this purpose, OMASES-VSA does not resort to (repeated or continuation) power flows, but rather simulates the time response of the system to a ramp increase in demand and/or generation. Thus, for a load increase, the demand coefficient $P_{i}^{o}$ of the load model shown in Fig. 1 is increased linearly with time according to:

$$
P_{i}^{o}(t)=P_{i}^{o}(0)+a_{i} g_{P} t \quad \text { with } \sum_{i} a_{i}=1
$$

where $a_{i}$ is a dimensionless participation factor and $g_{P}$ is the power increase gradient (MW/s), at system level. This parameter is chosen small enough to consider that the system evolves through steady states, and large enough to save computing time. The same relationship applies to reactive power. All LTCs, OELs, etc. respond with their delays to this increase in demand. Note that in the absence of corrective control, the total load power increase over a period $\Delta t$ is smaller than $g_{P} \Delta t$ under the effect of LTC deadbands and possibly limits.

Similarly, the active power setpoints of generators can be increased with time. The imbalances between load and generation due to losses and the above mentionned LTC effects, are covered by primary frequency (governor) control. To this purpose the frequency deviation is computed at each time step of the QSS simulation. The presence of this extra variable is balanced by the slack-bus phase angle relationship $\theta_{\text {slack }}=0$ as explained in [8].

Loadability limits are computed in the base case configuration, as well as for a limited set of dangerous contingencies, whose identification is explained in the next sections. The later limits are referred to as Post-Contingency Loadability limits (PCLL). To this purpose, in the same run, the contingency is simulated over a specified time interval, before the ramp increase in demand and generation is applied.

Besides power limits, the main outcome of such a simulation comes in the form of familiar PV curves. To this purpose, voltages at key buses are recorded as a function of the total load (power consumption, not demand) in various predefined areas.

\subsection{Secure Operation Limits}

For a given direction of stress, the Secure Operation Limit (SOL) corresponds to the most stressed operating point such that the system can withstand any contingency of a specified list [10]. Unlike a PCLL, this limit refers to the current, pre-contingency configuration of the system.

In the SOL computation, the stressed system states are obtained from a (pre-contingency) load flow computation, while the effect of a contingency is assessed using QSS simulation, initialised at the load flow solution.

The pre-contingency stress assumes full load recovery. Thus, at the $i$-th bus, the active and reactive powers increase according to:

$$
\begin{array}{ll}
P_{i}^{l}=P_{i}^{b c}+b_{i} S & \text { with } \sum_{i} b_{i}=1 \\
Q_{i}^{l}=Q_{i}^{b c}+c_{i} S & \text { with } \sum_{i} c_{i}=1
\end{array}
$$


where superscript $b c$ denotes base case values, $S$ is the total load power increase and $\left(b_{i}, c_{i}\right)$ are participation factors. The active power generation at the $j$-th bus is redispatched according to:

$$
P_{j}^{g}=P_{j}^{b c}+d_{j}(1+\epsilon) S \quad \text { with } \sum_{j} d_{j}=1
$$

where $d_{j}$ is a participation factor and $\epsilon$ accounts for changes in losses. The reactive power generations are rescheduled by letting the machines under AVR control, unless their reactive capabilities are reached.

The SOLs are determined by binary search. This simple and robust method consists of building smaller and smaller intervals $\left[S_{l} S_{u}\right]$ of stress values such that $S_{l}$ corresponds to an acceptable post-contingency evolution and $S_{u}$ to an unacceptable one. At each step, the interval is divided in two equal parts; if the midpoint is found acceptable (resp. unacceptable) it is taken as the new lower (resp. upper) bound. The procedure is repeated until $S_{u}-S_{l}$ is smaller than a specified tolerance $\Delta$.

To make the VSA function automatic, the binary search starts with $S_{l}=0$ (corresponding to base case) and

$$
S_{u}=\min \left(S_{\max }, f S_{N}\right)
$$

where $S_{\max }$ is the maximum stress of interest, $S_{N}$ the maximum stress that can be reached in the absence of contingency and $f$ is typically equal to 0.9 . $S_{N}$ is itself determined by binary search, considering an "empty" contingency.

To preserve computational efficiency in the presence of multiple contingencies, one should avoid repeating (pre-contingency) load flow computations relative to the same stress level. The number of load flows can be minimised by performing a Simultaneous Binary Search (SBS), which handles as many contingencies as possible at a given stress level. At a given step of this procedure, the unacceptable contingencies remaining from the previous step are simulated. If at least one of them is unacceptable, the acceptable ones are discarded (since their limits are higher than the current stress) and the search proceeds with the unacceptable ones only. For the most severe contingency(ies), the SOL is computed with the $\Delta$ accuracy, while for the others, a lower bound on the SOL is obtained. A simple example with 4 contingencies is shown in Fig. 2. The sequence of tested stresses is $S_{o}, S_{1}, S_{2}, S_{3}, S_{4}$. Five pre-contingency load flows are thus performed in the course of this SBS. The SOL corresponds to $S_{4}$ and relates to contingency no. 1. Contingency no. 4 is discarded at stress $S_{o}$, contingency no. 3 at $S_{1}$ and contingency no. 2 at $S_{3}$.

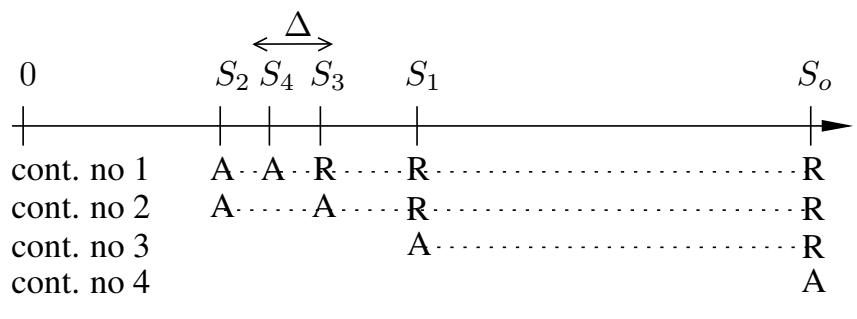

post-contingency evolution: $\mathrm{R}=$ refused $\mathrm{A}=$ refused

Figure 2: An example of simultaneous binary search

From there on, it is generally of interest to compute the individual SOLs of dangerous contingencies other than the most severe. Individual binary searches are performed to this purpose.

Note that the PCLLs and PV curves described in Section 4 are computed for the subset of contingencies found with the lowest margins at the end of the SOL determination (which is thus performed first). 
The distinction between SOLs and PCLLs is further discussed in Section 3.

\subsection{Contingency filtering}

Contingency filtering is an important step of any on-line security assessment application. A form of filtering takes place at the first step of the SBS, i.e. at maximum stress, when discarding the contingencies which yield an acceptable system response. However, in spite of the QSS simulation speed, it may take too long to simulate the system response to each contingency of a long list. An additional pre-filtering may be needed before the SBS is launched. In a majority of systems, post-contingency load flows can be advantageously used to this purpose.

Load flow equations with constant power loads and enforcement of generator reactive limits correspond to the long-term equilibrium that prevails after load voltage restoration by LTCs and machine excitation limitation by OELs. Insofar as voltage instability results from the loss of such an equilibrium, the corresponding load flow equations no longer have a solution and the NewtonRaphson algorithm diverges. On the other hand, divergence may result from purely numerical problems and some dynamic controls that help stability cannot be accounted for in the static load flow calculation. Conversely, some system dynamics may be responsible for an instability not detected by the load flow.

Keeping in mind the above conflicting aspects, the filtering consists of : (i) performing QSS simulation on a subset of contingencies labelled potentially harmful; (ii) identifying the latter as the contingencies that cause the load flow to diverge or some voltages to drop by more than a threshold $\Delta V$.

The value of $\Delta V$ must be taken large enough to filter out as many harmless contingencies as possible, but not too large to avoid missing a contingency with low SOL.

To make the filtering as reliable as possible, the post-contingency load flow data must closely match the QSS model. To this purpose, the reactive power limits of the generators are updated with their active power and terminal voltages, while active power imbalances are distributed over them according to frequency control.

\section{PCLL vs. SOL in Load Power Space}

The notion of "margin" in multi-load systems may have different interpretations depending upon the method used, as well as upon the way loads are grouped together.

Figures 3 and 4 sketch the difference between SOL and PCLL using a typical sequence of events as seen in the load power space for a hypothetical system. In particular, the vertical axis corresponds to the power consumption of the area mostly affected by the contingency of concern, while the horizontal axis is showing the power consumed in the rest of the system.

Figure 3 relates to SOL computation. Point A corresponds to the base case operating point of the system (EMS current state), where security margins are assessed. The dashed line indicates the chosen direction of stress. Points B and C in Fig. 3 are stressed pre-contingency operating points, at which the system response to the contingency is checked during the binary search of the SOL.

Consider for instance system operation at point A. Due to load sensitivity to voltage, under the effect of the contingency, the power consumed by loads drops and the system operating point 


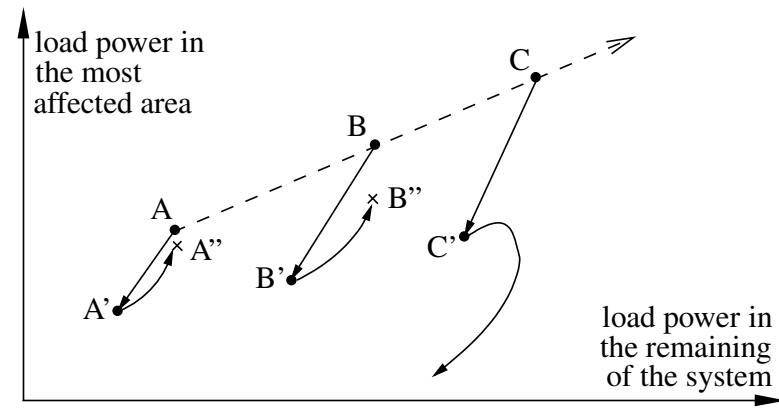

Figure 3: SOL computation in the load power space

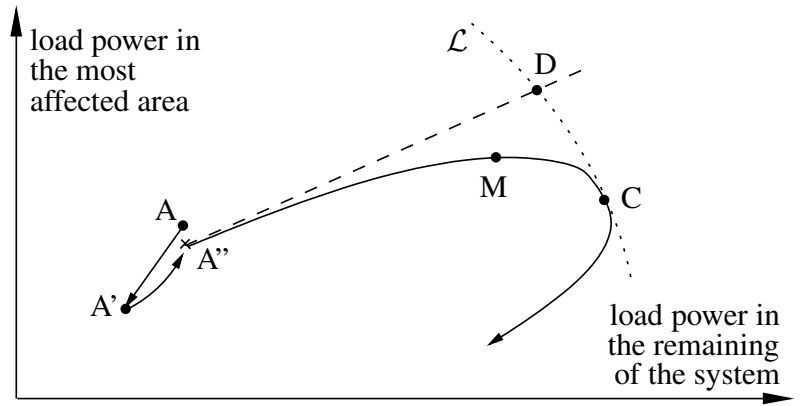

Figure 4: PCLL computation in the load power space

moves from A to A'. From there on load restoration mechanisms such as load tap changers bring the system back to operating point A" close to point A, but not exactly, due to LTC deadband effects. In stressed situations, it is possible that some taps reach their limits in the area affected by the contingency, in which case the corresponding load power will not be restored, as suggested by point B" in the same figure.

Tap limits have a stabilising effect. However, the final computed operating point is sensitive to the short-term load model used, which may not be known accurately. Moreover, in the real system, some other load restoration process can take place, not modelled here. In order to force load restoration and to avoid this "uncertain" system stabilisation, the range of tap changers can be artificially increased, so that limits are not met in a stable case. This option is offered in the software. Clearly, the criteria used to accept or reject a post-contingency evolution have to be adjusted accordingly.

Figure 4 relates to PCLL computation. Again, point A denotes the base case operating point. Following the contingency and the load restoration, the new operationg point is A", which differs from A for already mentioned reasons. From there on, a smooth ramp is imposed on the demand of voltage sensitive loads to bring the system to its loadability limit, and the LTCs are left to act. Under the effect of LTC deadbands, the system trajectory diverts from a straight line. Moreover, the figure refers to the situation where the LTCs in the affected area hit their limits. Under this effect, the distortion of stress direction becomes more pronounced, leaving the affected area with low distribution voltages.

The loadability surface $\mathcal{L}$ consists of all points where maximum power transfer conditions hold in the post-contingency configuration of the system. The post-contingency trajectory hits $\mathcal{L}$ at point $\mathrm{C}$, which is significantly different from point $\mathrm{D}$, the loadability limit under a linear load increase.

Note that along the post-contingency trajectory the power consumed in the affected area is maximum at point $\mathrm{M}$, which is not a loadability limit. At point $\mathrm{M}$ load restoration is still possible, but it 
is not achieved due to the combined effect of limited LTCs in the affected area and the continuing increase of the load in the rest of the system. The tip of the PV curve referring to this area will correspond to point $\mathrm{M}$ and demonstrates how far the load of the affected area will be able to restore following the contingency and assuming the specific stress direction.

It is clear that SOLs and PCLLs give different information and call for different interpretations.

SOL is better suited to quantify the "distance to insecurity" in terms of total system load. Along the pre-contingency stress we have normal operating points similar to those that operators are used to observe. A distance of 100 , or $1000 \mathrm{MW}$ in this respect is very meaningful to the operator who knows what is the increase he expects in the next hour or so.

The trajectory leading to a PCLL on the other hand, does not consist of "normal" operating points and the PCLL is not to be handled as a quantitative measure. Rather, it is valuable to demonstrate the system limits through local, regional, system-wide PV curves, which are convenient, widely accepted graphical representations. These post-contingency PV curves demonstrate graphically the amount of load restoration achieved in each area and in the whole system following the contingency (as well as the corresponding transmission voltage), and are thus providing useful information, complementary with respect to SOLs.

\section{Implementation aspects}

\subsection{Operating modes}

OMASES-VSA can be run in three modes: real-time, study and expert.

In real-time mode, the Energy Management System (EMS) feeds OMASES-VSA with network state estimator solutions. The execution of the VSA application is periodic and triggered automatically by the data transfer from the EMS. The periodicity of VSA execution is in the range of every 10-30 minutes. In the case of the Greek system (see next section), the whole VSA cycle takes 3 to 4 minutes on the average (on a Pentium IV PC).

In study mode, the VSA execution is launched manually by the user. The data used may be either real-time data or saved snapshots retrieved from the historical database by the user. A network solution stemming from the EMS state estimator or from a power flow program (saved cases) is thus retrieved from the database dedicated to this mode of operation.

Finally, in expert mode, the experienced user can have access to diagnosis tools, such as sensitivity and eigenvector analysis of Jacobian matrices [8]. In case of long-term voltage instability, an analysis of the long-term Jacobian allows to rank the bus active and reactive power injections by decreasing order of their effectiveness in counteracting the instability, thereby pointing out where generation should be rescheduled or committed, or load shed. When a singularity of the QSS time evolution has been met, corresponding to the loss of a short-term equilibrium, the short-term Jacobian is analysed to find which generators are mostly responsible for the loss of synchronism. The presentation of these linearisation-based analyses is out of scope of the present paper. 


\subsection{Overall architecture}

The OMASES platform relies on a Windows-2000 server hosting a relational database and an HTTP system, and connected through a Local Area Network (LAN) under the TCP/IP protocol. This server is separate from the mainframe computer which hosts the EMS platform and hierarchical databases. The communication between EMS and OMASES computers is accomplished with text files loaded by the EMS host onto the OMASES server. In the case of the HTSO control center, the OMASES server also runs the VSA computations.

Figure 5 outlines the overall architecture of the VSA application within the OMASES platform. The remaining of the description refers to the real-time mode. The other modes operate similarly.

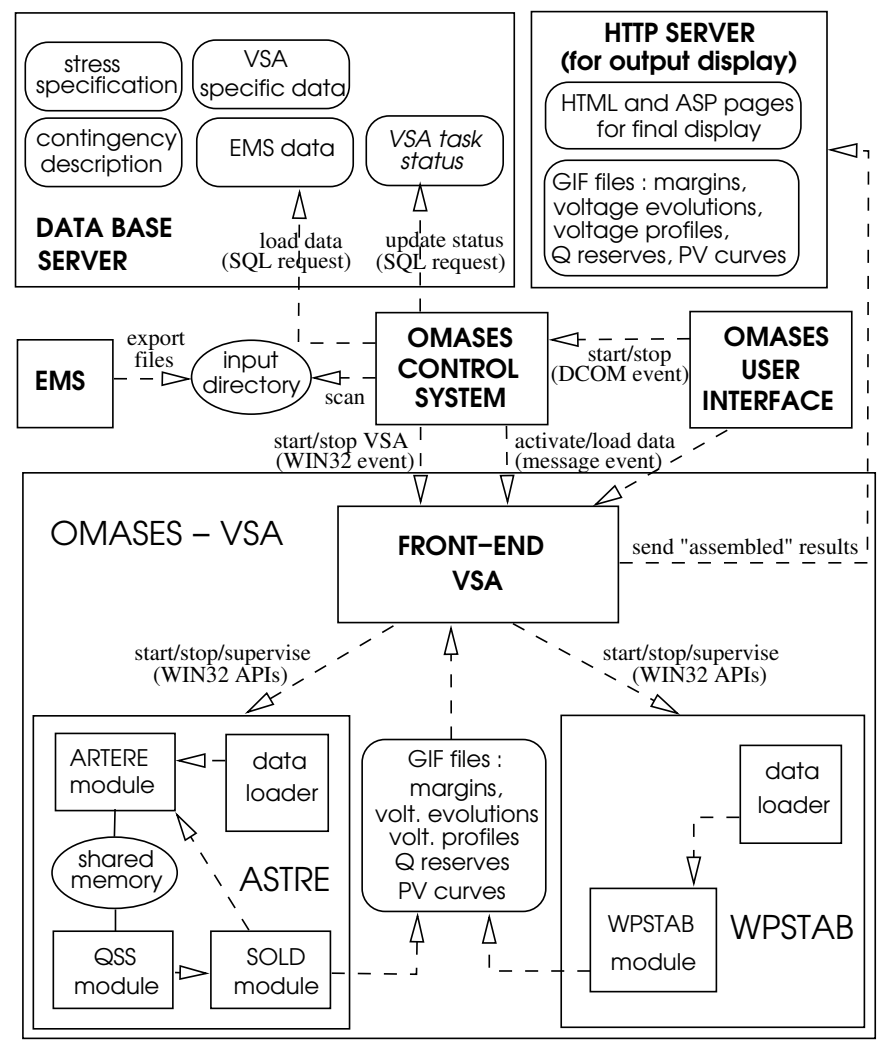

Figure 5: Architecture of VSA application within the OMASES platform

An EMS process periodically creates 14 text files containing the latest network solution computed by the state estimator. Each file is a description of a class of EMS components (e.g. substations, nodes, buses, lines, transformers, generators, loads, etc.) and corresponds to a table of the OMASES relational database. These files are sent by the same EMS process to a predefined real-time entry directory of the OMASES server, which triggers the VSA computation cycle.

The role of the OMASES Control System is to queue and dispatch OMASES messages to the VSA application function, detect the presence of new EMS files, load them into the database through a loader script, activate, synchronise and supervise the VSA task automatically. The other application functions are controlled in the same way.

The platform is controlled and the results are displayed through a Web interface.

The OMASES User Interface aims at starting and stopping the VSA task, activating it manually, and making its status visible to the OMASES user.

The VSA software itself is made up of two packages, developed prior to the project. 
The SOL computation and contingency filtering rely on the ASTRE software developed at the University of Liège and quoted in $[4,5,8,10]$. ASTRE itself is made up of a load flow module used for stressing the system and filtering contingencies, and a QSS simulator for simulating contingencies, both driven by a "conductor" module implementing the binary searches. The former two executables communicate through a shared memory for the data and through files for the outputs.

The computation of PCLLs and the preparation of PV-curves rely on the WPSTAB software developed at the National Technical University of Athens [11].

Within the context of the project, data loaders have been developed, whose role is to connect (through SQL requests) to the relational data base containing the EMS data, the VSA-specific data, the stress specification, the contingency description. The data loaders produce the ASCII files needed by ASTRE and WPSTAB, respectively.

A "front-end" program has been also developed in order to prepare, manage and remove dynamically the working environment of a new VSA session, communicate (through messages) with the remaining of the OMASES environment, launch the data loaders, call sequentially and supervise the ASTRE and WPSTAB programs.

The front-end also collects the GIF figures produced by ASTRE and WPSTAB and assemble them into HTML pages, together with ASP scripts, for WEB-based display by the HTTP server.

\section{VSA Analysis of the HTSO System}

\subsection{The Greek interconnected system}

The Greek transmission system serves the mainland of Greece and some interconnected islands. It consists of 400 and $150-\mathrm{kV}$ networks. The system is interconnected to the Balkan countries (Albania, Bulgaria, and FYROM) via three $400-\mathrm{kV}$ tie-lines, and to Italy via an asynchronous 400-kV AC-DC-AC link put in operation in 2002.

The main production center is in Northern Greece in the vicinity of a lignite rich area. The thermal power plants of this region produce about $70 \%$ of the total electricity of the Greek mainland. Significant hydro production exists in the North and Northwest of the country, while another lignite production area is in the Southern peninsula of Peloponnese.

On the other hand, the main consumption is in the metropolitan area of Athens. This leads to significant power transfers from North to South. Several critical operating conditions are associated with this geographical imbalance of generation and load especially in cases of reduced availability of the local generation in the Athens area and/or Peloponnese. These critical conditions are mainly linked to voltage problems following contingencies.

In this section, typical outputs of OMASES-VSA are presented for two snapshots of interest: a winter and a summer case, respectively. The winter case was obtained in real-time mode and corresponds to a relatively high load of the Greek system (but less than the Winter peak) that occurred on February 11, 2003. The summer case corresponds to the maximum system load for 2002, which occurred on July 16. It was produced in study mode from a saved EMS case (at that time, the platform was not yet installed at HTSO).

The stress considered for the VSA analysis is the default one, corresponding to a national load 
increase covered by the local generation reserve. Other system stresses, such as a load increase in the southern part of the system, a generation decrease in certain areas, or an increase of power flow from or to the external interconnections can be selected by the user, but are not considered in this paper.

The system model includes on the average 800 buses, 1100 branches and 110 generators. Seventy Greek generators are modelled taking into account saturation, AVR gain and governor speed droop effects, as detailed in [8]. The wind generation by induction machines is modelled as negative load. The same applies to the HVDC link with Italy. The external, equivalent generators are represented with constant voltage and participation to frequency control. The main discrete devices considered in QSS simulation are the LTCs on 400 transformers, the OXLs on the 70 detailed generators and turbine limiters on all of them.

The set of all N-1 contingencies and some probable N-2 ones are considered.

In SOL computations the range of LTCs controlling MV buses has been enlarged as explained in Section 3, while the normal ranges have been considered in PCLL computations.

\subsection{VSA Results}

The contingencies resulting in the most constraining SOLs for the Summer 2002 snapshot are listed in Table 1, together with the corresponding margins. The latter have been computed with a tolerance $\Delta=10 \mathrm{MW}$.

Table 1: SECURE OPERATION MARGINS (SUMMER CASE)
\begin{tabular}{|c|l|c|}
\hline no. & name & $\begin{array}{c}\text { margin } \\
(\mathrm{MW})\end{array}$ \\
\hline 329 & LINE_CON_TMOYnency & 0 \\
296 & LINE_CON_MOYPT-MESOG.1 & 0 \\
228 & LINE_CON_KQES-SXOLAP.1 & 0 \\
312 & LINE_CON_MESOG-KEPK2.1 & 0 \\
109 & LINE_CON_APG2-ARG1.1 & 73 \\
347 & LINE_CON_SXHM-QHBA.1 & 94 \\
69 & GEN_CON_K_LAVRIO.GFIC.UN & 146 \\
58 & GEN_CON_LAVRIO.GFIC.GEN2.UN & 167 \\
90 & LINE_CON_AGPAS-SKYDPA.1 & 292 \\
388 & LINE_CON_FILIP-AMFIPO.1 & 324 \\
22 & GEN_CON_AG.GEORG.GEN9.UN & 470 \\
all other contingencies & $>668$ \\
\hline
\end{tabular}

Note that the first four contingencies of Table 1 have a zero margin, meaning that the occurrence of any of these at the specific time the snapshot was taken, would result in unacceptable postcontingency conditions.

The Winter snapshot corresponds to a situation where one natural gas power station (AHSAG) in the Athens area was not in operation, which left the region with little reactive support. Although the total load was considerably lighter than that of the summer peak, the first contingency with system-wide effects (no. 61) has a very small margin, as seen in Table 2. In the same table, five contingencies appear with zero margin.

The contingencies with a small margin are not all equally dangerous. The extent of the problems caused to the system by an unacceptable contingency is easily assessed from the "voltage profiles" 


\begin{tabular}{|c|l|c|}
\hline no. & name & $\begin{array}{c}\text { margin } \\
(\mathrm{MW})\end{array}$ \\
\hline 254 & LINE_CON_KEPK2-KEPK1.1 & 0 \\
312 & LINE_CON_MESOG-KEPK2.1 & 0 \\
299 & LINE_CON_KARD-ELBA400.1 & 0 \\
296 & LINE_CON_MOYPT-MESOG.1 & 0 \\
228 & LINE_CON_KQES-SXOLAP.1 & 0 \\
61 & GEN_CON_K_LAVRIO.GFIC.UN & 40 \\
51 & GEN_CON_LAVRIO.GFIC.GEN2.UN & 79 \\
all other contingencies & $>316$ \\
\hline
\end{tabular}

automatically produced by the SOL computation. A voltage profile is obtained by taking a snapshot of the collapsing system at the marginally refused stress, and showing the number of buses with voltages below a certain level. The affected area is identified from the names of typical buses.

For instance, Fig. 6 shows the voltage profile corresponding to contingency No 329, the first one in Table 1. As can be seen, few buses with low voltages are affected. In the same way it is found out that all the contingencies with zero margin in Table 1, as well as the next two with a margin less than $100 \mathrm{MW}$, result in very local problems. The same holds true for the first five contingencies (with zero margin) of Table 2.

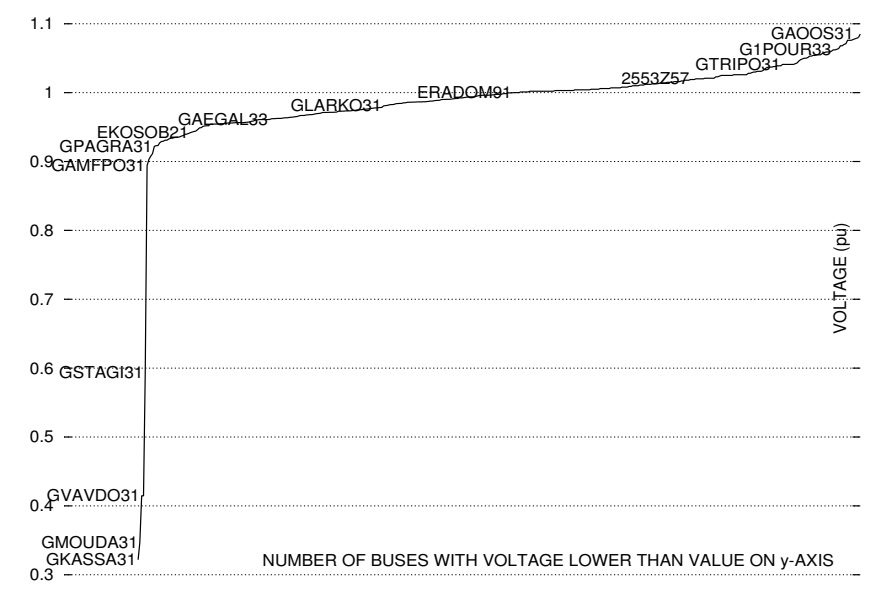

Figure 6: unstable voltage profile: contingency with very local impact

The nature of all these local problems is the breaking-up of a loop resulting in very long radial path to a relatively large load and, hence, in an excessive voltage drop. This situation was already known to HTSO engineers before it was confirmed by VSA analysis. Network reinforcements in all areas exhibiting this type of local voltage problems are under way.

In contrast, Fig. 7 shows the voltage profile for the first system-wide dangerous contingency of Table 1 (no. 69), namely the loss of the combined cycle plant in KLLavrio. As seen in this figure, the disturbance is widely spread, even though the focal point is in Central Greece just to the north of Athens metropolitan region. This picture is typical of a wide instability mode.

The same is true for contingency no. 61 of Table 2, which is again the loss of the combined cycle plant in K_Lavrio. The margin of this contingency is only 40 MW ! However, as this snapshot was taken at the daily peak and the load was already ramping down it was not deemed necessary to start the units in AHSAG.

Another output readily available for interpreting results is the post-contingency time evolution of 


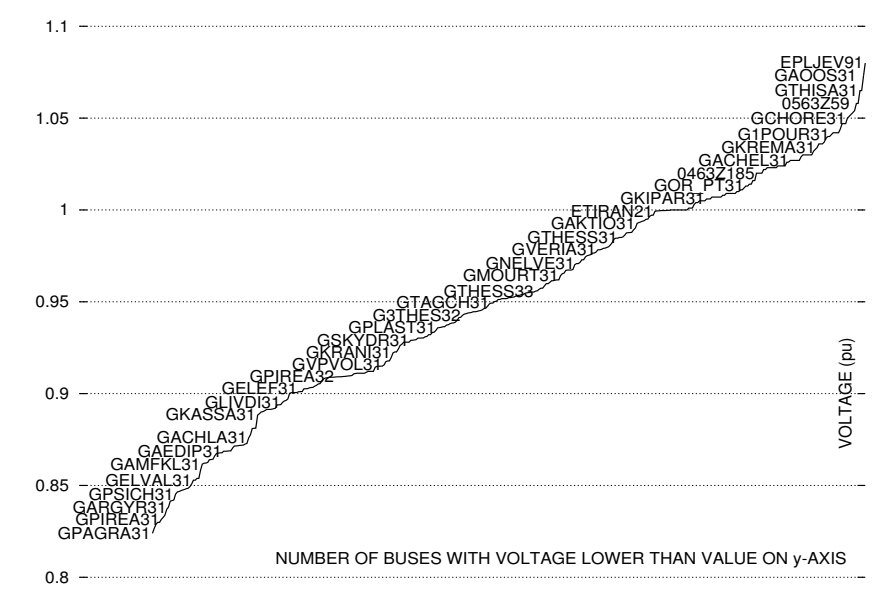

Figure 7: Unstable voltage profile: contingency with system-wide impact

the lowest transmission voltage at the marginally accepted and refused stresses, respectively. In the case of the Greek system, contingencies are simulated over a time interval of $900 \mathrm{~s}$, unless a singularity of the QSS simulation is met before, corresponding to loss of synchronism [8]. If not, the final voltages are compared to a lower acceptable value, typical of a partial voltage collapse (in this case $0.7 \mathrm{pu}$ ) to decide whether the system evolution is acceptable or not.

Figure 8 shows the voltage evolution for contingency no. 347 of Table 1. The contingency is applied at $t=10 \mathrm{~s}$. As can be seen, the marginally refused case does not correspond to a voltage instability but merely to final voltages that are a little below the 0.7 pu threshold. Hence, for this contingency, the SOL should not be interpreted as a stability limit.

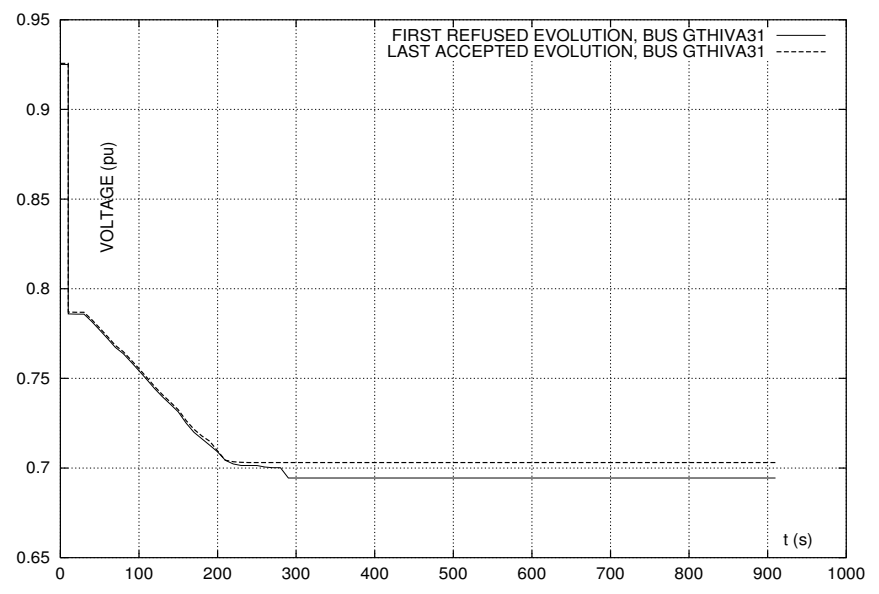

Figure 8: Post-contingency voltage evolutions: low but stable voltages

A quite different situation is shown in Fig. 9, relative to contingency no. 61 of Table 2. Here, the marginally unstable simulation ends up in a collapse and loss of synchronism at $t=880$ s. This evolution is very different from that of Fig. 8.

The diagnosis provided by OMASES-VSA in expert mode indicates that the loss of synchronism occurs at the Aliveri power station. This is confirmed by Fig. 10, showing the rotor angle curves of the two generators of this station as well as that of a generator in Northern Greece, for comparison purposes.

Figures 6 to 9 illustrate the type of information that can be retrieved from unstable post-contingency system evolutions obtained by QSS simulation. In the case of Figs. 7 and 9, a standard load flow 


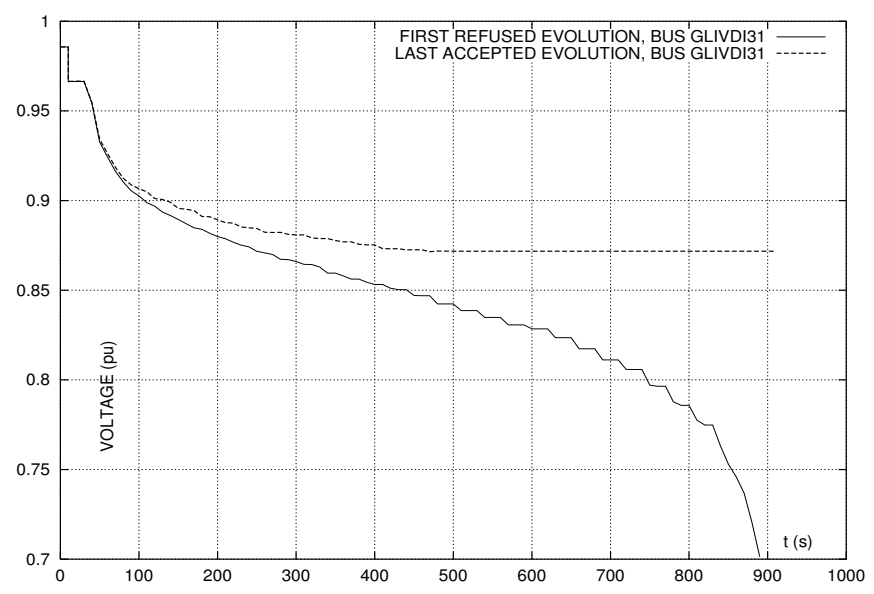

Figure 9: Post-contingency voltage evolution: voltage collapse

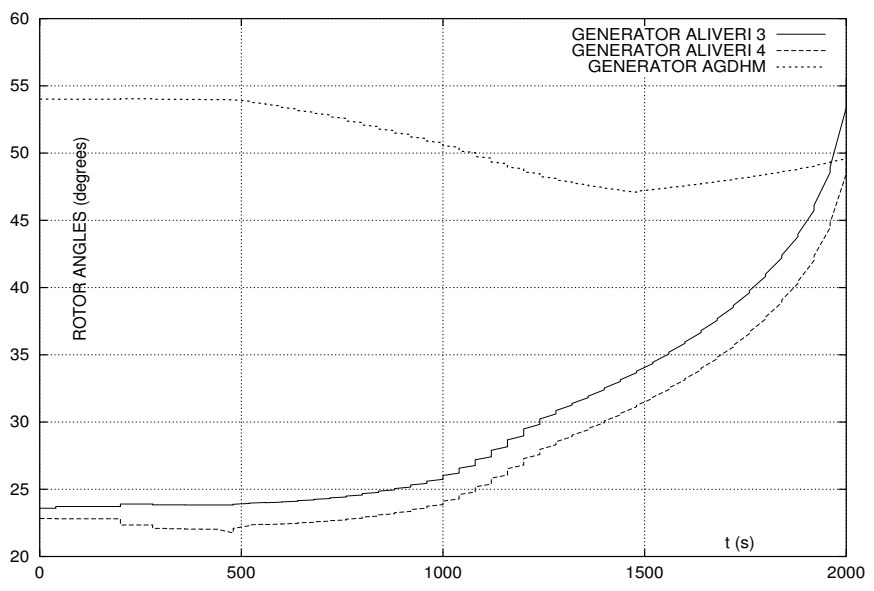

Figure 10: Evolution of rotor angles: conting. no. 61 followed by load increase

would have merely diverged, providing no further information.

The next four figures show (national or regional) PV curves obtained during loadability limit determination, as explained in Section 2.3.

Figure 11.a for Summer 2002 corresponds to the total system load and has been obtained by simply increasing the load demand in the base case. This result tells the operator how far the system can go even without contingencies. As seen from the figure, the load could increase by more than $500 \mathrm{MW}$ in the absence of any contingency.

PCLLs are obtained by stressing the system after contingencies. For instance, Figs. 11.b to d correspond to the system evolution under the effect of contingency no. 69 of Table 1 (first critical, loss of K_Lavrio), followed by a ramp increase in demand.

Figure 11.b confirms that this is a very severe contingency: the total load is barely able to restore its pre-contingency consumption even after the demand ramp. This is due to the exhaustion of the regulating range of the LTCs in the most affected area, as well as in some substations of the Athens region. At these places the power received by consumers is reduced due to low distribution voltages. Note that, as explained in Section 3, the PCLL implied in Fig. 11.b should not be compared to the SOL margin of Table 1, for which the stress is applied prior to the contingency assuming full restoration of load power. 

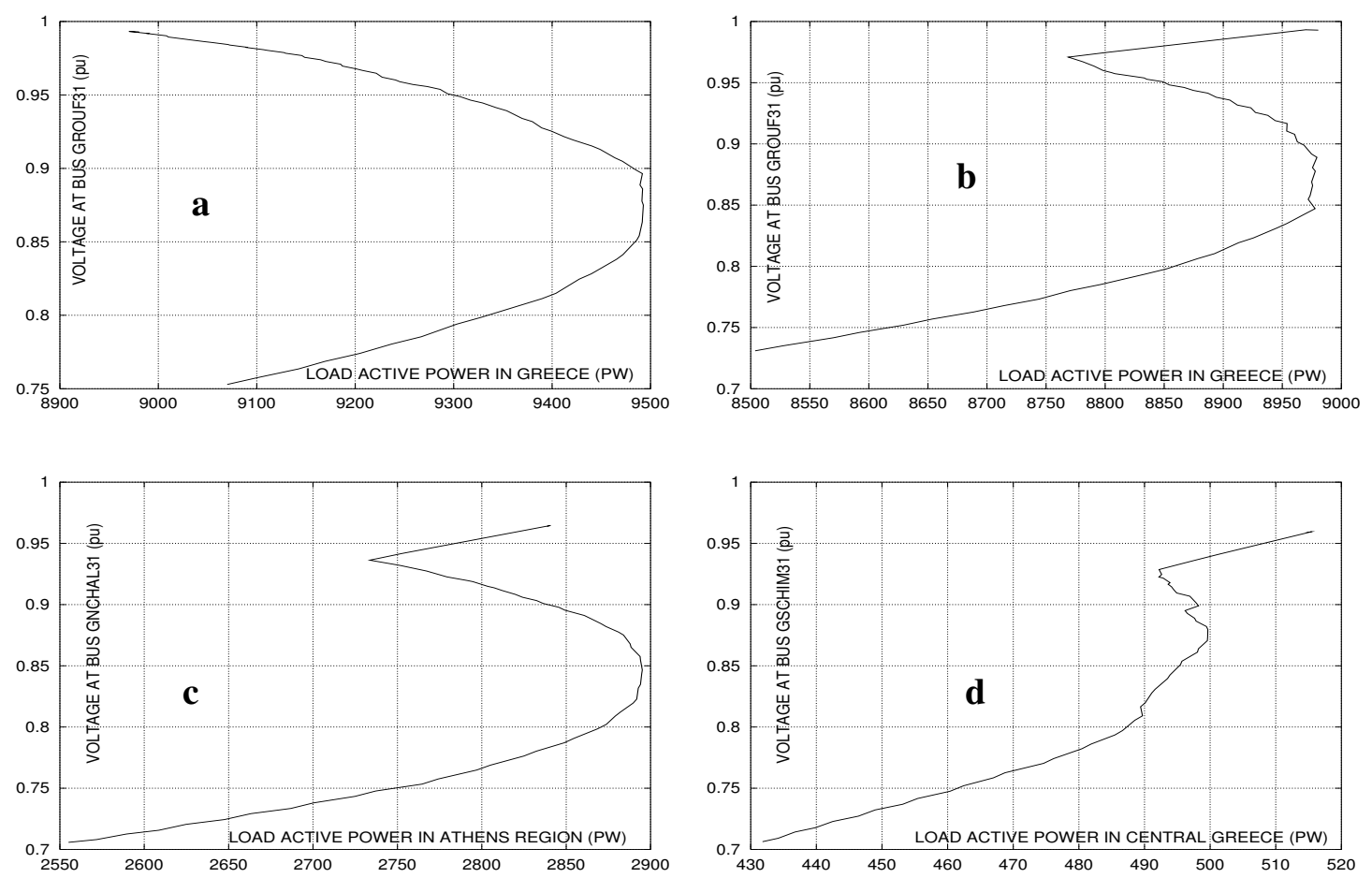

Figure 11: PV curves : (a) non contingency; (b-d) after contingency no. 69

The PV curve of Fig. 11.c, relative to the Athens region, shows that the latter is able to restore and even increase its load, despite the low voltages at certain transmission buses. The PV curve of the mostly affected area of Central Greece is shown in Fig. 11.d. Clearly, in this case, the load power is severely reduced after the contingency, as the increase in national load demand cannot lead to increased consumption in this area. Clearly, customer service in this area will greatly suffer in the event of such a contingency. HTSO is already planning network reinforcements and new capacitive compensation installation in the affected area.

The above results regarding the area mostly affected were confirmed also in the Winter 2003 case.

We conclude this section by a brief report on contingency filtering. As explained in Section 2.5, the system is stressed at the level given by (5) and a post-contingency load flow is run for each contingency. In the Summer 2002 case, for instance, the load is increased by $668 \mathrm{MW}$. At this load level, 309 contingencies out of 369 are declared harmless and are discarded. Among the 60 remaining contingencies, 50 lead to load flow divergence and 10 to a voltage drop of transmission voltages larger than the $\Delta V$ threshold, which has been set to $0.10 \mathrm{pu}$. These 60 potentially dangerous contingencies are analysed with QSS simulation (at the first step of the SBS) which identifies the 11 contingencies listed in Table 1 as causing real problems. The remaining 49 contingencies correspond to false alarms, of which 39 are caused by load flow divergence. The limit search proceeds with the 11 harmful contingencies.

\section{Conclusion}

This paper has described the advanced on-line VSA application developed within the context of the EU-sponsored project OMASES and implemented, tested and tuned at the Greek national control center of HTSO. Voltage security results obtained from this on-site experimentation have been also reported. 
The every-day performance of the tool has confirmed the reliability of the implemented methods and their compatibility with the constraints of a real-time environment.

The use of the on-line VSA tool during the testing period has demonstrated some, already known, local voltage problems. This served as an indirect validation and helped to increase the credibility of the tool.

The main benefit expected by HTSO from such a tool is the possibility to estimate security limits more accurately than by steady-state tools and operate closer to them. The use of traditional static security analysis in the Hellenic Interconnected system leads sometimes to either start expensive generation in the area of Athens or shed loads while it is not necessary. Such a situation has been avoided during the Winter of 2003 during the test phase of the tool. Therefore, the latter is also expected to facilitate the every-day operation of the system, as well as avoid unnecessary uplift costs.

Finally, the use of the on-line VSA tool will contribute to the training of the operators, will allow a better understanding of voltage instability phenomena and will make it easier to evaluate the benefit of countermeasures against credible disturbances.

\section{Acknowledgments}

The OMASES project was supported by European Commission under contract No ENK6-CT2000-000064. The contributions of Gaëtan Hassé in Liège and George Manos in Athens to the software development phase for the on-line application is duly acknowledged, as well as discussions with Florin Capitanescu about the methods. Thanks are due to the whole OMASES team, especially the Alstom group, who led and coordinated the project.

\section{References}

[1] C.W. Taylor, Power system voltage stability, EPRI Power System Engineering Series, Mc Graw-Hill, 1994

[2] B.Gao, G.K. Morison, P. Kundur, "Towards the development of a systematic approach for voltage stability assessment of large-scale power systems", IEEE Trans. on Power Systems, Vol. 11, pp. 1314- 1324, 1996

[3] C.W. Taylor, "The future in on-line security assessment and wide-area stability control", Proc. IEEE PES Winter Meeting 2000, vol. 1, pp. 78-83, January 2000

[4] "Voltage Stability Assessment: concepts, practices and tools", Special publication of the IEEE Power System Stability Subcommittee (C. Cañizares, Editor), 2002, ISBN 0780378695

[5] "Techniques for power system stability limit search", IEEE PES special publication IEEE TP-138-0, Jan. 2000

[6] A. Bihain, G. Burt, F. Casamata, T. Koronides, R. Lopez, S. Massucco, D. Ruiz-Vega, C. Vournas, "Advance Perspectives and Implementation of Dynamic Security Assessment in the Open Market Environment”, paper 39-101, CIGRE 2002 
[7] A. Bihain, D. Cirio, M. Fiorina, R. Lopez, D. Lucarella, S. Massucco, D. Ruiz Vega, C. Vournas, T. Van Cutsem, L. Wehenkel, "OMASES: A Dynamic Security Assessment Tool for the New Market Environment”, IEE/PES Bologna Power Tech, June 2003

[8] T. Van Cutsem, C. Vournas, Voltage Stability of Electric Power Systems, Boston, Kluwer Academic Publishers, 1998

[9] C. Vournas, G. Manos, J. Kabouris, T. Van Cutsem, "Analysis of a voltage instability incident in the Greek power system", Proc. IEEE PES Winter Meeting 2000, vol. 2, pp. 1483-1488, Jan 2000

[10] T. Van Cutsem, C. Moisse, R. Mailhot, "Determination of secure operating limits with respect to voltage collapse", IEEE Trans. on Power Systems, vol. 14, 1999, pp. 327-335

[11] C. Vournas, Scientific Co-ordinator, "Software Development for Voltage Stability Analysis", Project 96 SYN 95, NTUA, Greece 